\title{
Initial treatment of macular oedema due to central retinal vein occlusion-which anti-VEGF agent to choose?
}

\author{
Elad Moisseiev ${ }^{1,2} \cdot$ Anat Loewenstein ${ }^{1,3}$
}

Received: 24 September 2019 / Accepted: 25 September 2019 / Published online: 26 November 2019

(c) The Royal College of Ophthalmologists 2019

Central Retinal Vein Occlusion (CRVO) is the second most common retinal vascular disease (the first being diabetic retinopathy), and is associated with cystoid macular oedema (CMO) that causes vision loss $[1,2]$. Intravitreal injections of anti-vascular endothelial growth factor (VEGF) agents are the mainstay of treatment for CMO due to CRVO, but although each of the available agents (ranibizumab, bevacizumab and aflibercept) has demonstrated high efficacy and excellent safety profiles for the treatment of this common condition, very few comparative trials have been published regarding these agents.

The SCORE2 study, published in 2017 [3], compared bevacizumab and aflibercept for the treatment of CMO due to CRVO. It included 362 patients who were randomized 1:1 and received 6 monthly injections of either agent. At 6 months, the mean improvement in visual acuity (VA) was 18.6 letters in the bevacizumab group and 18.9 letters in the aflibercept group, demonstrating that bevacizumab is non-inferior to aflibercept for the treatment of this condition.

Last month, the results of the LEAVO study were published [4]. This trial compared all three anti-VEGF agents (bevacizumab, ranibizumab and aflibercept) for the treatment of CMO due to CRVO, and included 463 patients who were randomized 1:1:1. Patients in all groups received four mandatory monthly injections, and thereafter retreatment was based on specific pre-defined criteria, and were followed for 2 years. At 2 years, the mean improvement in VA was 12.5 letters for ranibizumab, 15.1 letters for aflibercept,

Elad Moisseiev

elad_moi@netvision.net.il

1 Sackler School of Medicine, Tel Aviv University, Tel Aviv, Israel

2 Department of Ophthalmology, Meir Medical Center, Kfar Saba, Israel

3 Department of Ophthalmology, Tel Aviv Medical Center, Tel Aviv, Israel and 9.8 letters for bevacizumab. Fewer injections were administered over the 2 years in the aflibercept group than in the ranibizumab and bevacizumab groups (mean of $10 \mathrm{vs}$ 11.8 and 11.5 , respectively). It should also be noted that the rates of more than three lines of VA improvement were similar in all groups $(47 \%, 52 \%$ and $45 \%$ for ranibizumab, aflibercept and bevacizumab, respectively), and that no differences in safety were noted between groups. Specific comparisons using intention-to-treat adjusted mean BCVA difference showed that aflibercept demonstrated non-inferiority compared to ranibizumab, but that bevacizumab was not non-inferior to either ranibizumab or aflibercept.

Although these results did not demonstrate that bevacizumab is significantly worse for the treatment of CMO due to CRVO, they were inconclusive regarding the VA changes at 2 years, and raised the possibility that the choice of the initial anti-VEGF agent may determine the long-term outcomes of treatment.

Thus, we have somewhat different outcomes from two well-designed clinical trials, SCORE2 and LEAVO. This can be explained by significant differences between these two trials. First, the SCORE2 study was only 6 months long, while LEAVO was 2 years. The difference between drugs may manifest itself only after a longer time interval. Second, patients received monthly injections in SCORE2, while in LEAVO after the first four injections monitoring was every 4-8 weeks and re-injections were determined by specific criteria, and patients received injections in longer intervals in this study. Thus, in SCORE2 patients received more intensive therapy during the first 6 months. The difference between drugs maybe smaller is the drugs are used in a very intensive manner. Third, the SCORE2 study included patients with hemi-retinal vein occlusion (HRVO), which has a better prognosis, while LEAVO did not. Fourth, LEAVO included patients with better initial VA (upper limit of 78 letters compared to 73 in SCORE2), which may have caused a ceiling effect in the VA improvement. 
In many countries, mostly due to economic reasons, bevacizumab is commonly used as the initial anti-VEGF agent for the treatment of CMO due to CRVO, and realworld results from recent tears have indicated good outcome $[5,6]$. It is possible that with different monitoring and injection frequencies, the results would have been closer for all three anti-VEGF agents in the LEAVO trial. Still, it seems that for the regimen used in the LEAVO trial which is the most common regimen used, although good outcome was achieved with all three anti-VEGF drugs available today, the use of bevacizumab was not non-inferior to ranibizumab and aflibercept, a fact that needs to be considered when it is given as first line to our patients.

\section{Compliance with ethical standards}

Conflict of interest None of the authors have any proprietary interest in this publication.

Publisher's note Springer Nature remains neutral with regard to jurisdictional claims in published maps and institutional affiliations.

\section{References}

1. The Eye Disease Case-Control Study Group. Risk factors for central retinal vein occlusion. Arch Ophthalmol. 1996;114:545-54.

2. The Central Vein Occlusion Study Group Natural history and clinical management of central retinal vein occlusion. Arch Ophthalmol. 1997;115:486-91.

3. Scott IU, VanVeldhuisen PC, Ip MS, Blodi BA, Oden NL, Awh $\mathrm{CC}$, et al. SCORE2 Investigator Group. Effect of bevacizumab vs aflibercept on visual acuity among patients with macular edema due to central retinal vein occlusion: the SCORE2 randomized clinical trial. JAMA. 2017;317:2072-87.

4. Hykin P, Prevost AT, Vasconcelos JC, Murphy C, Kelly J, Ramu J, et al. LEAVO Study Group. Clinical effectiveness of intravitreal therapy with ranibizumab vs aflibercept vs bevacizumab for macular edema secondary to central retinal vein occlusion: a randomized clinical trial. JAMA Ophthalmol. 2019. [Epub ahead of print].

5. Jumper JM, Dugel PU, Chen S, Blinder KJ, Walt JG. Anti-VEGF treatment of macular edema associated with retinal vein occlusion: patterns of use and effectiveness in clinical practice (ECHO study report 2). Clin Ophthalmol. 2018;3:621-9.

6. Wecker T, Ehlken C, Bühler A, Lange C, Agostini H, Böhringer D, et al. Five-year visual acuity outcomes and injection patterns in patients with pro-re-nata treatments for AMD, DME, RVO and myopic CNV. Br J Ophthalmol. 2017;101:353-9. 